\title{
Neue Ergebnisse quartärgeologischer Untersuchungen im Gebiet der "Falkenberg-Endmoräne"
}

\author{
Hans Dietrich LanG*)
}

Drenthe main advance, ground moraine, end moraine, geological structure, kames, Falkenberg end moraine, lower saxony

\begin{abstract}
Kurzfassung: Neuere Untersuchungen und Bohrungen haben die vom Autor im Jahre 1983 mitgeteilten Vorstellungen über den quartärgeologischen Aufbau dieses Gebietes (LANG 1983 a) bestätigt und erweitert.

Die zum Drenthe-Hauptvorstoß gehörende Grundmoräne taucht unter die Endmoränenbögen im Gebiet um den Falken-Berg ab und ist auch darunter erbohrt worden. Auf den Endmoränenrücken fanden sich keinerlei Reste von Grundmoräne, desgleichen waren keine Hinweise auf Stauchungen zu beobachten. Aus Bohrungen ergeben sich keine Hinweise auf präexistente Aufragungen im Untergrund. Die Rücken der verschiedenen Endmoränenbögen sind als Satzendmoränen aufzufassen, die nicht mehr vom nordischen Inlandeis überfahren wurden.
\end{abstract}

Der Mühlenberg-Zug, der Tannensieksberg und ein kleiner Rücken wenig südwestlich davon werden als Kames gedeutet. Auch sie werden, wie Bohrungen zeigten, von der Grundmoräne des Drenthe-Hauptvorstoßes unterlagert.

Die Endmoränen entstanden durch zwei von NW und von NE bis NNE vorstoßende Gletscherloben, die hier aufeinandertrafen und danach abtauten. Die Endmoränen und die Kames sind dem ausgehenden Drenthe-Stadium der Saale-Kaltzeit zuzurechnen.

\section{[New Results of Quaternary-Geological Research in the "Falken Berg" End Moraine Area]}

\begin{abstract}
New field research and drilling have confirmed a conception of the Quaternary-Geological structure of the "Falken Berg" area in extension of results published by the author in 1983 (LANG 1983 a).

The fact that the ground moraine belonging to the Drenthe main advance is dipping under the end moraine ridges in the Falken Berg area has been proved by drilling. On the end moraine ridges relics of the ground moraine never have been found; likewise no references to pushings have been observed. Equally drilling holes have not proved any pre-existing towering in the underground. The ridges of the several arcuated end moraines have been interpreted as recessional end moraines.
\end{abstract}

The author explaines the Mühlenberg Zug, the Tannensieksberg and a small ridge not far in the south-west of it as

*) Anschrift des Verfassers: Dr. H. D. LANG, Marienburger Weg 9, 30916 Isernhagen NB.
Kames. As proved by drillings they are underlayn by the ground moraine of the Drenthe main advance.

The end moraines were shaped by two ice lobes that advanced from NE and from NE to NNE and hit each other at that point, melting down after that. The origin of the end moraines and the Kames can be located at the late Drenthe Stadium of the Saale glaciation.

\section{Einleitung}

Im Jahre 1983 habe ich im 33. Band dieser Zeitschrift über "Aufbau, Alter und regionale Einordnung der Endmoränen im Gebiet des Falkenberges bei Fallingbostel (Niedersachsen)" berichtet (LANG 1983 a). Entgegen der damaligen Annahme war es mir in den Jahren 1985 bis 1987 doch noch möglich, im Rahmen der geologischen Landesaufnahme das Blatt 3125 Bergen der top. Karte 1:25 000 geologisch zu bearbeiten, für das bisher nur eine geologische Übersichtsaufnahme vorlag (NIEDERMAYER 1950 b).

Auf dem Blatt Bergen liegt der Zentralteil der "F a lkenberg-Endmoräne", der etwas mehr als das nordwestliche Viertel des Blattgebietes umfaßt. Er ist durch eine breite Senke, in der das "Große Moor" liegt, vom Mühlenberg-Zug getrennt, der etwa aus der nordöstlichen Blattecke in SSWRichtung bis in die Höhe von Bergen zieht und den WOLDSTEDT (1939) "als einen Gegenflügel der Falkenberg-Endmoräne" angesehen hatte. Die Kartierarbeiten haben eine Reihe neuer Ergebnisse zum quartär-geologischen Aufbau dieses Gebietes erbracht. Als besonders wichtig haben sich die Ergebnisse der bis zu $50 \mathrm{~m}$ tiefen Bohrungen mit einem Drillbohrgerät des Niedersächsischen Landesamtes für Bodenforschungen im Zuge der Kartierarbeiten erwiesen.

Die Ergebnisse werden hier in Form eines Nachtrages zu der Arbeit des Autors von 1983 vorgestellt. Zum Verständnis des Textes ist es nötig, Passagen aus der Arbeit LANG 1983 a zu wiederholen, desgleichen einige Literaturzitate. 


\section{Die Endmoränen in der Umgebung des Falkenberges}

STOller (1918), der Erstbearbeiter dieses Gebietes, beschrieb in seinem Geologischen Führer durch die Lüneburger Heide das Gebiet um den fast $150 \mathrm{~m}$ üb. NN aufragenden Falkenberg als ein "... geschlossenes Massiv aus der Ebene aufsteigender Endmoränen ...", die er seiner vorletzten Eiszeit zuordnete. WoldstedT (1938) führte den Begriff "FalkenbergEndmoräne" in das Schrifttum ein und sah darin die Hauptfortsetzung des Rehburger Stadiums nördlich der Aller. Ein saalezeitlicher Gletscher, so schrieb WOLDSTEDT im Jahre 1939, der durch norwegisches Eis nach Süden abgedrängt wurde, habe die Moränen aufgestaucht. In einer Arbeit von 1950 stellte WolDSTEDT die Falkenberg-Endmoräne in seine Soltauer Staffel, die jüngste der drei Phasen des Drenthe-Stadiums der Saale-Eiszeit. LüTTIG (1964) sah in seiner Falkenberg-Staffel eine ältere Staffel der Northeimer Phase, die er etwas jünger einstufte als die zur gleichen Phase gehörende Ostenholzer Staffel. Duphorn (in WOLDSTEDT 1974) folgte in groben Zügen dieser Vorstellung und sah in der Fal-
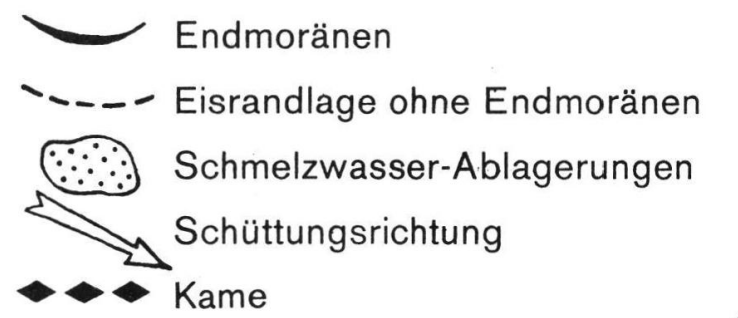

Fallingbostel

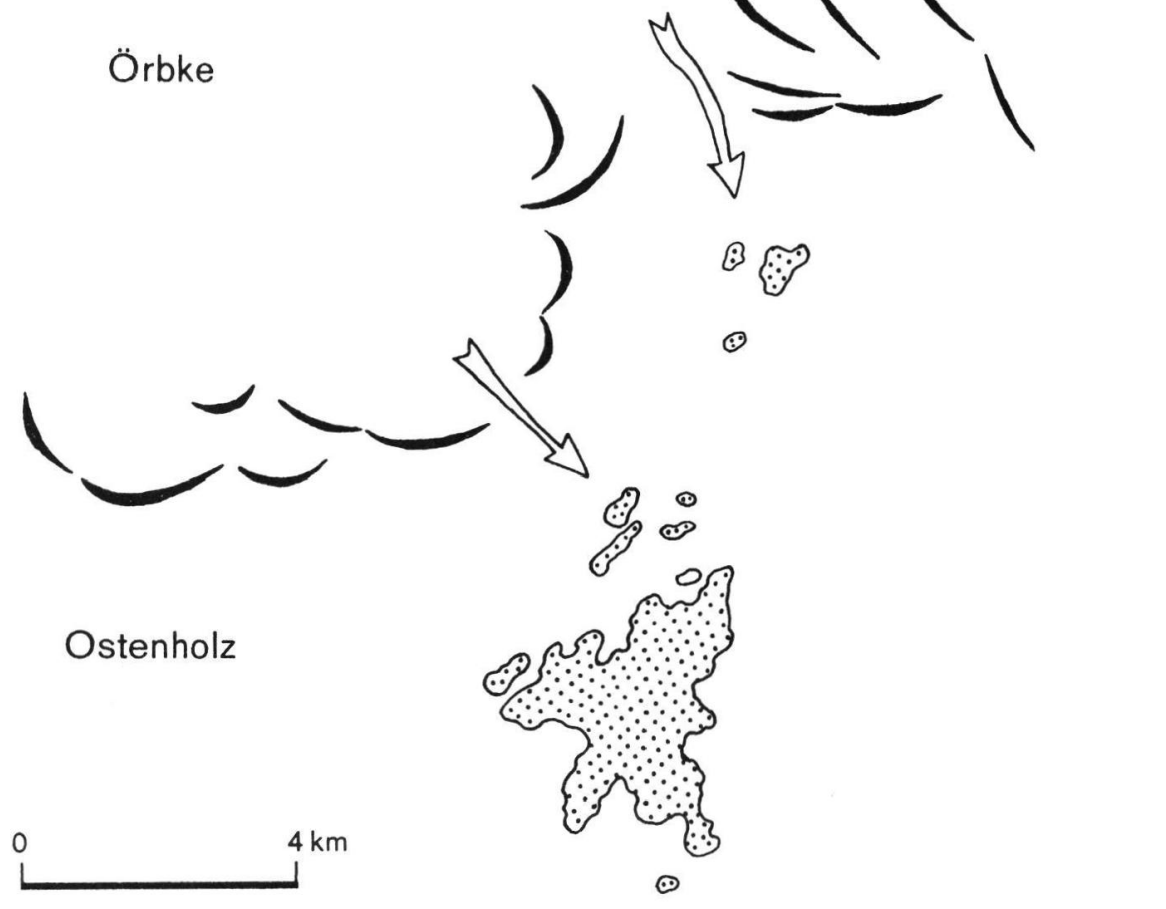


kenberg-Endmoräne eine frühdrenthestadiale, vom nordischen Inlandeis überfahrene Endmoräne.

Wie bei LANG (1983 a) dargestellt, sind im Gebiet um den Falkenberg drei voneinander getrennte, meist mehrfach hintereinander gestaffelte bogige Endmoränen zu unterscheiden (s. Abb. 1):

\section{der Örbker Endmoränenbogen}

der Kl. Bockeler Endmoränenbogen

der Becklinger Endmoränenbogen.

Durch die Achterberg-Senke, die den Örbker Endmoränenbogen von den beiden nördlich bzw. nordöstlich davon gelegenen Kl. Bockeler und Becklinger Endmoränenbögen trennt, und durch eine Scharte im Örbker Endmoränenbogen, die heute der Hohebach durchfließt, dürften die Schmelzwässer nach S und nach SE abgeflossen sein. Sie haben dabei einen Sander aufgeschüttet, der sich bis nahe an die Aller-Niederung heran verfolgen läßt (LANG 1980, 1983 a, 1983 b).

Der fast "aus einem Guß" bestehende Örbker Endmoränenbogen erreicht im GoldbockenBerg eine Höhe von 121,9 m üb. NN und überragt sein Hinterland um etwa $45 \mathrm{~m}$. Dort steht weitflächig Grundmoräne zu Tage an (Niedermayer $1950 \mathrm{a}$ ), die jedoch nicht, wie nach einer älteren Bohrung anzunehmen (s. LANG 1983 a), zweigeteilt ist. Sie wird nach neueren Bohrungen bis etwa $20 \mathrm{~m}$ mächtig und besteht in den oberen Metern aus Geschiebelehm, in den unteren aus Geschiebemergel.

Zwei Bohrungen auf der Endmoräne selbst nahe dem Goldbocken-Berg und nahe dem Hamm-Berg haben eine Wechselfolge von Grob-, Mittel- und Feinsanden erbohrt, Grundmoräne aber nicht angetroffen. Niedermayer (1950 a) gibt jedoch auf seiner Karte ein kleines Vorkommen von Grundmoräne unter etwa $1 \mathrm{~m}$ Sandüberdeckung vom östlichen Abfall des Örbker Endmoränenbogens etwa 1,5 km östlich des Goldbocken-Berges an, in einer Geländehöhe von 86 bis $87 \mathrm{~m}$ üb. NN. Vermutlich handelt es sich hier um die gleiche Grundmoräne, die nördlich des Endmoränenbogens in seinem Hinterland ansteht und die hier unter dünner Sandüberdeckung auftaucht. Auf der Endmoräne selbst, die auffallend frische Formen zeigt (NIEDERMAYER 1950 a; LANG 1983 a), sind bisher keinerlei Reste von Grundmoräne gefunden worden.

Aus dem Gebiet des K1. Bockeler Endmoräne n bogens, auf Blatt 3124 Fallingbostel gelegen (Niedermayer 1950 b) liegen keine neuen Befunde gegenüber 1983 vor.

Der aus einer Reihe einzelner Staffeln bestehende Becklinger Endmoränenbogen mit dem 149,6 m hohen Falken-Berg nimmt den Großteil des Hochgebietes im nordwestlichen Viertel des Blattes Bergen ein. Die einzelnen zu diesem Bogen gehörenden Rücken sind leicht gebogen und nach $\mathrm{N}$ bzw. NE geöffnet. Nach $\mathrm{N}$ geht dieses reich gegliederte Gebiet in eine pultartige, leicht nach NE abfallende Hochfläche über (LANG 1983 a).

Die einzelnen Rücken bestehen in ihrem tieferen Teil aus z. T. grobsandigen Fein- und Mittelsanden, im oberen Teil aus einer Folge von bis zu 20 - 30 m mächtigem Grobsand und Kies. Die gesamte Abfolge kann bis etwa $70 \mathrm{~m}$ mächtig werden. Die Täler zwischen den einzelnen Rücken dürften durch die nach-drenthezeitliche Erosion stark überprägt und vertieft worden sein. Die grobsandig-kiesigen Deckschichten auf den Rücken haben der Erosion einen größeren Widerstand entgegengesetzt als die sandigen Schichten in den ursprünglich vermutlich nur flachen Dellen zwischen den Rücken und haben eine flächenhafte Erosion verhindert. Vermutlich haben diese frischen Formen die früheren Bearbeiter der Endmoränen im Falkenberg-Gebiet dazu gebracht, diese Rücken als durch Stauchung entstanden anzusehen.

An keiner Stelle im Hochgebiet um den Falken-Berg und nördlich davon fanden sich Reste von Grundmoräne. Lediglich vereinzelte größere Geschiebe bis zu etwa $1 \mathrm{~m}^{3}$ Größe kommen vor. Desgleichen haben sich an keiner Stelle Lagerungsstörungen finden lassen, die als Ergebnis einer Stauchung durch das Inlandeis gedeutet werden könnten.

Im südlichen und östlichen Vorland des Becklinger Endmoränenbogens treten einzelne Grundmoränenflecken oder, wie nördlich der nach Wietzendorf-Soltau führenden Bahnstrecke, zwischen Kl. Amerika und Öhus, größere Grundmoränenflächen auf. Bohrungen im nördlichen Randgebiet des Blattes Bergen der GK 25 in der Nähe der neuen Straße nach Soltau (Bohrungen Nr. G 9 und H 10, Archiv NLfB, s. auch LANG 1989) haben gezeigt, daß die Grundmoräne unter die Sande und Kiese der Endmoränen abtaucht. Auch im Bruchkamp, wenig östlich vom Meyerhof nahe Bleckmar, war die Überlagerung von Geschiebelehm durch Sande der Endmoränen im Bohrstock zu beobachten.

Die Bohrung G 5 (Archiv NLfB), etwa $4 \mathrm{~km}$ westlich Becklingen gelegen, also im zentralen Teil des Hochgebietes mit den Endmoränen, hat unter 10,5 m mächtigen Sanden und Kiesen der Endmoränen 2,5 m mächtigen Geschiebelehm des Drenthe-Stadiums nachgewiesen. Andere Bohrungen haben in vergleichbarer Höhenlage unter Ausfall des Geschiebelehms graue schluffige Tone oder $z$. T. leuchtend gelbbraune Schluffe des Drenthe-Stadiums, die das Liegende der Grundmoräne bilden (LANG 1989), teilweise auch mit einer Steinsohle, nachgewiesen. Diese Grenzfläche zwischen den Sanden und Kiesen der Endmoränen und der drenthestadialen Grund- 
moräne, bei ihrem Fehlen der unmittelbar unter der Grundmoräne folgenden Schichten, läßt sich in einer Höhe von etwa $80 \mathrm{~m}$ üb. NN relativ gut verfolgen. Dieses Niveau entspricht etwa der Höhenlage der Oberkante der drenthestadialen Grundmoräne im westlichen und südlichen Vorland des Becklinger Endmoränenbogens. Damit wird die Vermutung von Schucht (1935), “... daß sich in diesem diluvialen Hochgebiet präexistente Reliefformen widerspiegeln ...," durch Bohrungen widerlegt.

Diese Befunde untermauern die schon bei LANG (1983 a) vorgenommene Einstufung des Örbker Endmoränenbogens und der Endmoränen im Hochgebiet um den Falkenberg in eine späte Phase des Drenthe-Stadiums. Sie sind jünger als die drenthestadiale Hauptmoräne, der sie aufgesetzt sind. Zusammen mit der Frische der Oberflächenformen, besonders des Örbker Endmoränenbogens, weisen alle Beobachtungen darauf hin, daß es sich hier um Satzendmoränen handelt, die nicht mehr vom Inlandeis überfahren wurden.

\section{Mühlenberg-Zug und Tannensieksberg}

Als einen östlichen Gegenflügel der FalkenbergEndmoräne faßte WOLDSTEDT (1939) in seiner Arbeit "Über Endmoränen in der südlichen Lüneburger Heide" den Mühlenberg-Zug auf, der sich etwa von der Wietze bei Wietzendorf bis in die Höhe von Bergen verfolgen läßt. Dieser von NNE nach SSW ziehende Rücken ist etwa $11 \mathrm{~km}$ lang und an seinem nördlichen Ende 1 - 1,2 km breit, an seinem südlichen etwa $2 \mathrm{~km}$. Seine größten Höhen erreicht er im Bleckmarsberg mit 95,4 $\mathrm{m}$ üb. NN und im namengebenden Mühlenberg bei Widdernhausen mit 92,7 m üb. NN. Er überragt seine Umgebung um durchschnittlich 15 - $20 \mathrm{~m}$. Vom Hochgebiet mit dem Becklinger Endmoränenbogen trennt ihn eine etwa $4 \mathrm{~km}$ breite und sich nach $\mathrm{S}$ auf etwa $1 \mathrm{~km}$ verschmälernde Senke, die weitgehend vom "Großen Moor" eingenommen wird (s. Abb. 1).

Der Mühlenberg-Zug wird von etwa 10 - 15 m mächtigen Mittel- und Grobsanden mit Feinsandlagen aufgebaut, denen auf seinen höheren Teilen 5- $7 \mathrm{~m}$ mächtige Deckschichten aus Grobsand und Kies aufgelagert sind. Seine südliche Hälfte wird von Sandlöß überlagert, der bis etwa $2 \mathrm{~m}$ mächtig wird (LANG 1989, 1990). Eine bestimmte Korngrößenabfolge innerhalb der Sedimente dieses Rückens ist, abgesehen von den gorbsandig-kiesigen Deckschichten, nicht erkennbar.

Geschiebelehm tritt auf dem Mühlenberg-Zug nicht auf, er kommt aber auf größeren Flächen zu beiden Seiten des Rückens vor. Im Verlaufe der Kartierarbeiten hat sich gezeigt, daß dieser Geschiebelehm unter den Rücken abtaucht. Die Bohrungen G 18 (etwa $2,3 \mathrm{~km}$ südlich Marbostel) und G 21 (etwa $1,5 \mathrm{~km}$ südlich Bleckmar; Archiv. Nieder. L.-Amt f. Bodenforsch.), beide auf dem Rücken angesetzt, haben $2 \mathrm{~m}$ bzw. 4,5 m mächtigen Geschiebelehm an seiner Basis erschlossen. Der Rücken ist also einer Grundmoräne aufgesetzt, die, wie in einer Kiesgrube nahe Häger Döpe unweit Bergen zu beobachten war, gestauchte Sande und Kiese überlagert und die der Drenthe-Hauptmoräne zuzuordnen ist (LANG 1983 b).

Schotteraufsammlungen bei Marbostel und in einer Grube bei Bleckmar zeigen, daß hier nur nordisches Material vorkommt. In Aufschlüssen auf dem Mühlenberg-Zug (Sandgrube etwa $800 \mathrm{~m}$ östlich Marbostel; Alte Sandgrube auf dem Sittern-Berg; Sandgrube an der Bahnlinie Bergen-Bleckmar, etwa $1 \mathrm{~km}$ südöstlich Bleckmar) waren keine Hinweise auf durch Stauchung hervorgerufene Lagerungsstörungen zu beobachten. Die aufgeschlossenen Sande sind relativ sauber geschichtet und zeigen Schrägschichtung. Sowohl in der alten, heute teilweise verfüllten Sandgrube auf dem Sittern-Berg (R 35 64 950, H 5857 550, 36 Messungen) als auch in der Sandgrube an der Bahnlinie Bergen-Bleckmar (R 3563 600, H 5854 940, 66 Messungen) zeigen die Maxima der Einfallstichtung der schräggeschichteten Sande und Kiese nach SW, nur wenig abweichend von der Streichrichtung des Mühlenberg-Zuges (s. Abb. 2).

Ähnlich gebaute Rücken, im wesentlichen aus Sand bestehend, mit grobsandig-kiesigen Deckschichten, sind der Tannensieksberg und ein kleiner Rücken wenig südwestlich davon (der auf den Karten keinen Eigennamen führt), denen die Bahnstrecke von Bleckmar nach Wietzendorf folgt. Sie werden bei einer Breite von etwa $300 \mathrm{~m}$ ungefähr 900 bzw. $700 \mathrm{~m}$ lang und erheben sich 5 - $10 \mathrm{~m}$ über ihre weitgehend vermoorte Umgebung. Sie werden von der hier weitflächig auftretenden drenthestadialen Grundmoräne unterlagert (LANG 1989).

Woldstedt (1939) sah im Mühlenberg-Zug einen Teil der Falkenberg-Endmoräne, LüTTIG (1964: Taf. 7) deutete ihn als Os.

Die Breite des Mühlenberg-Zuges zwischen 1,2 und etwa $2 \mathrm{~km}$ bei einer Länge von etwa $11 \mathrm{~km}$, der fast gerade Verlauf, die (soweit aufgeschlossen) relativ ungestörten Schichten und vor allen Dingen die weitgehend der Längserstreckung des Rückens entsprechenden Maxima der Einfallsrichtung der Schrägschichtung dürften eher zu einem $\mathrm{Ka}$ me als zu einem Os passen. Das gleiche wird für den Tannensieksberg und den kleineren Rücken wenig südwestlich davon angenommen. Diese drei Rücken entstanden nahe dem Eisrand, zur Zeit des Zerfalls 

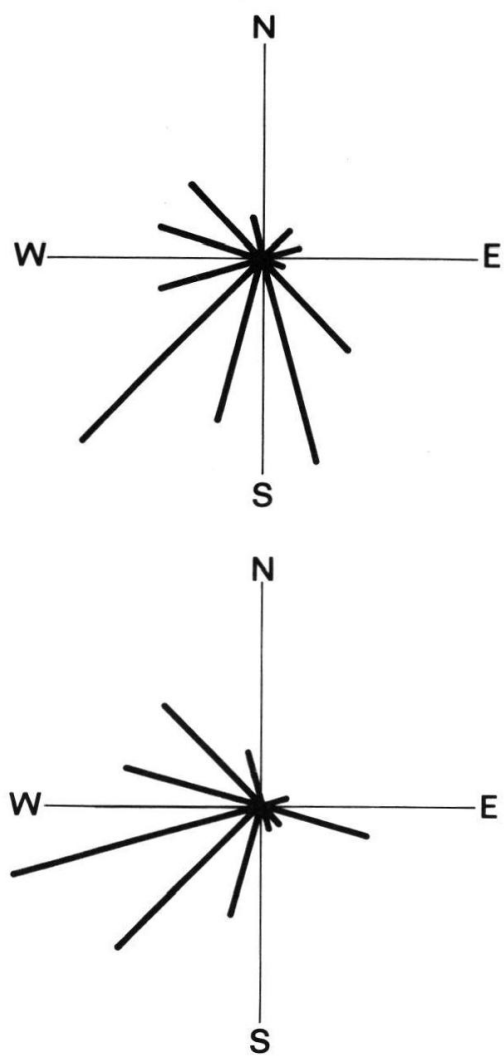

Abb. 2: Die Schüttungsrichtung von Sanden und Kiesen des Mühlenberg-Zuges

oben: Sandgrube etwa $1 \mathrm{~km}$ südöstlich Bleckmar (R 3563 600, H 5854 940, 66 Messungen)

unten: Sandgrube auf dem Sittern-Berg, etwa $1 \mathrm{~km}$ nordwestlich Nindorf (R 3564 950, H 5857 550, 36 Messungen)

des Eislobus, der den Becklinger Endmoränenbogen gebildet hat. Aus nördlicher bzw. nordöstlicher Richtung kommendes Schmelzwasser, das in Richtung des Eisrandes abfloß, dürfte diese Rücken zwischen stehengebliebenen Eisklötzen abgelagert haben. Der unmittelbar am Eisrand sich verbreiternde Abstand zwischen den Eisklötzen könnte bedingt haben, daß das Kames sich an seinem Südrand verbreitert und auseinanderfließt.

Die Kames dürften also etwas jünger als die Endmoränen selbst sein.

\section{Regionalgeologische und zeitliche Einordnung}

Die früher als ein einheitlicher Komplex angesehene Falkenberg-Endmoräne (WoldstedT 1939) besteht aus 3 Endmoränenbögen, die aus mehrfach hintereinander gestaffelten Rücken bestehen und denen im Süden ein Sander vorgelagert ist, der bis an die Aller-Niederung heranreicht. Die drenthestadiale Haupt-Grundmoräne, die diesen Sander unterlagert, steht im südlichen und östlichen Randgebiet des vornehmlich dem Becklinger Endmoränenbogen zuzuordnenden Hochgebiet um den Falkenberg zu Tage an und taucht, wie es auch Bohrungen nachgewiesen haben, darunter ab. Sander und Endmoränen sind also nachweislich jünger als der Drenthe-Hauptvorstoß.

Zwei von NNW und von NE bis NNE vorstoßende Gletscherloben haben das Untersuchungsgebiet nicht streng gleichzeitig erreicht. Der von NW kommende Gletscherlobus ist etwas vorangeeilt, und vor seiner Stirn konnte sich eine fast modellhaft deutliche Satz-Endmoräne entwickeln. Vermutlich erst danach traf der "leicht nachhinkende" östliche Gletscherlobus schräg auf den westlichen Gletscherlobus, der ihn bremste und daran hinderte, weiter nach Süden vorzustoßen. Die vergleichsweise vielen, hintereinander gestaffelten Rücken des Becklinger Endmoränenbogens sind vermutlich darauf zurückzuführen, daß es diesem Gletscherlobus an dem gehörigen Platz zur Ausbildung eines breit ausladenden Endmoränenbogens fehlte. Der kl. Bockeler Endmoränenbogen auf der westlichen Seite mit seinen kurzen, nach NW geöffneten Rücken ist vermutlich auch dem Örbker Endmoränenbogen zuzuschreiben; er ist jünger als der Örbker Endmoränenbogen und zeigt vielleicht schon ein Abschmelzstadium an.

Wie die Bohrungen nachgewiesen haben, gab es keine prä-existenten Aufragungen im Untergrund, die die Lage der Endmoränenbögen bestimmt haben könnten.

Das Aufeinandertreffen zweier sich schräg aufeinander zubewegenden Eisloben, also rein lokale und nicht vom Untergrund abhängige Gegebenheiten, haben die Lage der Endmoränen um den Falkenberg herum bedingt. Sie erklären vielleicht auch die Schwierigkeit, eine Fortsetzung der Endmoränen nach Westen und Osten zu finden. Mit den bisher bekannten, sicher aus dem ausgehenden DrentheStadium stammenden und also nicht vom Inlandeis überfahrenen Endmoränen in der weiteren Umgebung läßt sich bis heute keine glaubhafte Verbindung herstellen.

Mühlenberg, Tannensieksberg und der kleine Rücken wenig südwestlich davon, die als Kames gedeutet werden, gehören bereits in die Abschmelzphase der Gletscherloben. Sie entstanden vermutlich nahe dem Außenrand des nun im Zerfall begriffenen östlichen der beiden Gletscherloben, in breiten Lücken zwischen noch stehengebliebenen Eisklötzen. Es ist denkbar, daß das Südende des Mühlenberg-Zuges, das wesentlich 
breiter als sein Nordende ist, bereits unmittelbar am Übergang zum eisfreien Gebiet oder schon im eisfreien Bereich entstanden ist.

\section{Schriftenverzeichnis}

LaNG, H. D. (1980): Geol. Karte Niedersachsen 1:25 000, Erl. Bl. 3224 Westenholz: 98 S., 12 Abb., 5 Tab., 5 Kt.; Hannover (Nieders. L.-Amt Bodenforsch.)

- (1983 a): Aufbau, Alter und regionale Einordnung der Endmoränen im Gebiet des Falken-Berges bei Fallingbostel (Niedersachsen). - Eiszeitalter u. Gegenwart, 33: 31 - 43, 3 Abb., 1 Tab.; Hannover.

- $\quad$ (1983 b): Geol. Karte Niedersachsen 1 : 25 000, Erl. Bl. 3225 Offen: 108 S., 8 Abb., 5 Tab., 8 Kt.; Hannover (Nieders. L.-Amt Bodenforsch.)

- (1990): Der Sandlöß in der Umgebung von Bergen Krs. Celle - Verbreitung, Zusammensetzung und Entstehung - . - Eiszeitalter u. Gegenwart, 40: 97 - 106, 3 Abb.; Hannover.

- (1992): Geol. Karte Niedersachsen 1:25 000, Erl. Bl. 3125 Bergen: 100 S., 11 Abb., 9 Tab., 7 Kt.; Hannover (Nieders. L.-Amt Bodenforsch.)
LütTiG, G. (1966): Prinzipielles zur Quartärstratigraphie. Geol. Jb., 82: 177 - 202, 1 Abb., 1 Taf.; Hannover. [Sonderdrucke 1964.]

Niedermayer, J. (1950 a): Erläuterungen für das Meßtischblatt Fallingbostel 1:25 000: 8 S, 1 Kt.; Archiv Nieders. L.-Amt Bodenforsch. Hannover. - [Unveröff.]

- (1950 b): Erläuterungen zu Blatt Bergen 1 : 25 000: 9 S., 1 Kt.; Archiv Nieders. L.-Amt Bodenforsch. Hannover. - [Unveröff.]

STOLler, J.. (1918): Geologischer Führer durch die Lüneburger Heide. - 168 S., 38 Fig., 8 Taf.; Braunschweig (Vieweg).

WOLDSTEDT, P. (1938): Über Vorstoß- und Rückzugsfronten des Inlandeises in Norddeutschland. - Geol. Rdsch. 29,6: 481 - 490, 2 Abb.; Stuttgart.

- (1939): Über Endmoränen in der südlichen Lüneburger Heide. - Abh. Naturw. Ver. Bremen, 31: 236 - 246, 3 Abb.; Bremen.

- (1974): Norddeutschland und angrenzende Gebiete im Eiszeitalter. 3. Aufl., neu bearb. u. herausgegeb. von K. DupHORN: 500 S., 91 Abb., 26 Tab.; Stuttgart (Koehler).

Manuskript eingegangen am 6. 4. 1992 